\title{
Lebesgue Constants and Optimal Node Systems via Symbolic Computations
}

\author{
Short Paper \\ Robert Vajda \\ University of Szeged, Szeged, Hungary \\ vajdar@math.u-szeged.hu
}

\begin{abstract}
Polynomial interpolation is a classical method to approximate continuous functions by polynomials. To measure the correctness of the approximation, Lebesgue constants are introduced. For a given node system $X^{(n+1)}=\left\{x_{1}<\ldots<x_{n+1}\right\}\left(x_{j} \in[a, b]\right)$, the Lebesgue function $\lambda_{n}(x)$ is the sum of the modulus of the Lagrange basis polynomials built on $X^{(n+1)}$. The Lebesgue constant $\Lambda_{n}$ assigned to the function $\lambda_{n}(x)$ is its maximum over $[a, b]$. The Lebesgue constant bounds the interpolation error, i.e., the interpolation polynomial is at most $\left(1+\Lambda_{n}\right)$ times worse then the best approximation. The minimum of the $\Lambda_{n}$ 's for fixed $n$ and interval $[a, b]$ is called the optimal Lebesgue constant $\Lambda_{n}^{*}$. For specific interpolation node systems such as the equidistant system, numerical results for the Lebesgue constants $\Lambda_{n}$ and their asymptotic behavior are known [3, 7]. However, to give explicit symbolic expression for the minimal Lebesgue constant $\Lambda_{n}^{*}$ is computationally difficult. In this work, motivated by Rack [5, 6, we are interested for expressing the minimal Lebesgue constants symbolically on $[-1,1]$ and we are also looking for the characterization of the those node systems which realize the minimal Lebesgue constants. We exploited the equioscillation property of the Lebesgue function [4] and used quantifier elimination and Groebner Basis as tools [1, 2]. Most of the computation is done in Mathematica [8].
\end{abstract}

Acknowledgement. The research of the author was partially supported by the HSRF (OTKA), grant number K83219.

\section{References}

[1] D. S. Arnon - G. E. Collins - S. McCallum, Cylindrical Algebraic Decomposition I: The Basic Algorithm. In: Caviness-Johnson (eds): Quantifier Elimination and Cylindrical Algebraic Decomposition, 136-151, Springer, 1998.

[2] B. Buchberger, Ein Algorithmus zum Auffinden der Basiselemente des Restklassenringes nach einem nulldimensionalen Polynomideal (An algorithm for finding the basis elements in the residue class ring modulo a zero dimensional polynomial ideal). PhD Thesis, Innsbruck, 1965.

[3] J. S. Hesthaven, From electrostatics to almost optimal nodal sets for polynomial interpolation in a simplex, SIAM J. Numer. Anal., Vol. 35, No. 2, 655-676, 1998.

[4] T. A. Kilgore, A characterization of the Lagrange interpolating projection with minimal Tchebycheff norm, J. Approx. Theory 24 (1978), no. 4, 273-288.

[5] H.-J. Rack, An example of optimal nodes for interpolation, International Journal of Mathematical Education in Science and Technology 15 (3): 355-357, 1984.

[6] H.-J. Rack, An example of optimal nodes for interpolation revisited, Advances in Applied Mathematics and Approximation Theory, Springer Proceedings in Mathematics \& Statistics, Volume 41, 117-120, 2013.

[7] S. J. Simon, Lebesgue constants in polynomial interpolation, Annales Mathematicae et Informaticae 33: 109-123, 2006.

[8] Wolfram Research Inc., Mathematica, Version 9.0, Champaign, Illinois, 2012. 